\title{
Emodin attenuates calcium overload and endoplasmic reticulum stress in AR42J rat pancreatic acinar cells
}

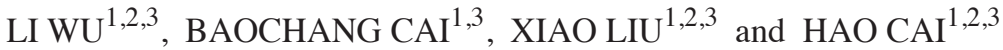 \\ ${ }^{1}$ National First-Class Key Discipline for Science of Chinese Materia Medica; \\ ${ }^{2}$ College of Pharmacy, Nanjing University of Chinese Medicine; ${ }^{3}$ Engineering Center, \\ State Ministry of Education for Standardization of Chinese Medicine Processing, \\ Nanjing University of Chinese Medicine, Nanjing, Jiangsu 210023, P.R. China
}

Received April 10,2013; Accepted October 25, 2013

DOI: $10.3892 / \mathrm{mmr} .2013 .1773$

\begin{abstract}
The aim of the present study was to investigate the protective effects of emodin against calcium overload and endoplasmic reticulum (ER) stress in an acute pancreatitis model in vitro. AR42J rat pancreatic acinar cells treated with cerulein $\left(10^{-7} \mathrm{M}\right)$ and lipoplysaccharide (LPS; $\left.10 \mathrm{mg} / \mathrm{l}\right)$ were used to mimic acute pancreatitis in vitro. The amylase activity in cellular lysates and culture media was detected by spectrophotometry. The level of cytosolic calcium was measured by laser confocal microscopy. Cell apoptosis and necrosis were examined by flow cytometry. Reverse transcription-polymerase chain reaction was used to determine the mRNA expression of ER chaperone immunoglobulin-binding protein (Bip) and downstream molecules, including protein kinase-like ER kinase (PERK), activation transcription factor 6 (ATF6) and inositol-requiring protein 1 (IRE1). The results showed that emodin significantly reduced the expression and release of amylase, attenuated calcium overload and decreased the mRNA expression of Bip, PERK, ATF6 and IRE1 which was significantly elevated in AR42J cells treated with cerulein and LPS. Emodin also reduced cell apoptosis and necrosis. Therefore, the results of the present study indicate that emodin protects against AR42J cell injury caused by cerulein and LPS. These effects may be associated with reduced calcium overload and inhibited ER stress responses.
\end{abstract}

\section{Introduction}

Acute pancreatitis (AP) is a commonly occurring disease of considerable severity. AP involves a complicated cascade of events initiated by the injury of pancreatic acinar cells (1),

Correspondence to: Professor Baochang Cai or Dr Xiao Liu, College of Pharmacy, Nanjing University of Chinese Medicine, 138 Xianlin Avenue, Nanjing, Jiangsu 210023, P.R. China

E-mail: bccai@126.com

E-mail: liuxiao04_0@163.com

Key words: acute pancreatitis, calcium overload, endoplasmic reticulum stress, emodin resulting in local and systemic inflammation (2). Although the exact mechanism underlying acinar cell injury in AP is complex and remains to be defined, it has been hypothesized that calcium overload is the trigger of acinar cell injury in AP (3). Previous studies have indicated that excess insult agents, including bile salts (4), ethanol metabolites and fatty acids $(5,6)$, induce marked and global calcium release from the endoplasmic reticulum (ER) to the cytoplasm, which further induces premature intracellular digestive enzyme activation, vacuolization, secretory blockade, disorganization of the cytoskeleton and cellular necrosis. Furthermore, calcium depletion of the ER stores has been observed to induce the activation of ER stress responses, which is associated with acinar injury and AP (7). In rats with arginine-induced AP (8), specific key ER stress sensors, including RNA-activated protein kinase-like ER kinase (PERK), activation transcription factor 6 (ATF6) and inositol requiring protein 1 (IRE1), are highly activated and these signaling molecules lead to complex responses, including translation attenuation, inflammatory and immune responses and induction of apoptosis. Similarly, in the cerulein model of acute pancreatitis, ER stress responses are observed early, paralleling trypsinogen activation $(9,10)$. It has also been observed that stimulation of isolated pancreatic acini by secretagogues generates distinct ER stress responses and eventually cell death (11). Moreover, reducing ER stress by tauroursodeoxycholic acid, a chemical chaperone or by genetic manipulation of ER chaperone immunoglobulin-binding protein (Bip), induces protective effects against acute pancreatitis $(12,13)$. All these observations indicate that ER stress is one of the mechanisms underlying pancreatic acinar cell injury and may be a potential target for preventing and treating this disease.

Emodin (6-methyl-1,3,8-tanthragallol), the predominant effective component of rhubarb, has been used clinically to treat AP for a number of years in China (14). Several in vivo investigations have also shown that emodin has significant therapeutic effects on AP in rats by correcting intestinal flora disturbances, promoting intestinal peristalsis (15) and inhibiting inflammatory cytokine release and pancreatin activity (16). Furthermore, studies have shown that emodin ameliorates pancreatic acinar cell injury by suppressing toll-like receptor 4 expression, inhibiting pancreatin activity, scavenging oxygen free radicals, 
increasing occludin expression and promoting apoptosis in the pathogenesis of AP. Our previous studies have shown that emodin markedly ameliorates pancreatic injury via attenuating ER stress responses in the pancreas of rats with AP induced by sodium taurocholate (17). In the present study, the effects of emodin on the activation of ER stress sensor proteins were further evaluated using an in vitro model of AP.

\section{Materials and methods}

Materials. Rat pancreatic acinar AR42J cells (CRL 1492) were obtained from the American Type Culture Collection (Manassas, VA, USA). Emodin was purchased from China National Institutes for Food and Drug Control (Beijing, China). Ham's F12 medium, cerulein, lipoplysaccharide (LPS) and dexamethasone were purchased from Sigma-Aldrich, (St. Louis, MO, USA). Fetal bovine serum (FBS), glutamine, penicillin/streptomycin, fluo-3 acetoxymethyl ester were obtained from Invitrogen Life Technologies (Carlsbad, CA, USA). The amylase assay kit was purchased from Nanjing Jiancheng Bioengineering Institute (Nanjing, China). RNase-free DNase set and RNA safe were purchased from Tiangen Biotech. Co. Ltd. (Beijing, China). An RNA extraction kit, Oligo(dT) $)_{15}$ and dNTP were obtained from Servier Pharmaceutical Research \& Development Co. Ltd. (Beijing, China). M-MLV reverse transcriptase was purchased from Promega Corporation (Madison, WI, USA). Primers were designed and synthesized by Invitrogen Life Technologies.

Cell culture. AR42J is the only cell line that exhibits numerous characteristics of normal pancreatic acinar cells and has been used to study the secretion, growth and proliferation of exocrine pancreatic cells (18). In the present study, this cell line was used as an in vitro model to evaluate the effects of emodin against pancreatic acinar cell injury and the activation of ER stress sensor proteins. AR42J cells were cultured in Ham's F12 medium supplemented with $2 \mathrm{mM}$ glutamine, 20\% FBS and antibiotics (100 U/ml penicillin and $100 \mu \mathrm{g} / \mathrm{ml}$ streptomycin) at $37^{\circ} \mathrm{C}$ in a humidified atmosphere of $5 \% \mathrm{CO}_{2}$ and $95 \%$ air. Treatment with dexamethasone has been observed to convert these cells into exocrine cells (19). AR42J cells were plated at a density of $2 \times 10^{4}$ cells $/ \mathrm{cm}^{2}$ in 6 -well culture dishes, incubated for $48 \mathrm{~h}$ with $100 \mathrm{nM}$ dexamethasone and washed with phosphate-buffered saline (PBS) prior to incubation in fresh medium.

Emodin treatment protocol. In preliminary experiments, the toxic effects of emodin on AR42J cells were detected by examining the cellular viability and amylase activity in culture media. AR42J cells were incubated in the absence of stimulus or in the presence of 5, 10, 20,40 and $80 \mu \mathrm{M}$ emodin. Following incubation for 3, 6, 12, 24 and 48 h, the cell viability and amylase activity in the culture media were evaluated. The results showed that the cellular viability and amylase activity in the culture media did not vary in incubation with 5-40 $\mu \mathrm{M}$ emodin for $48 \mathrm{~h}$; however, the viability of AR42J cells was reduced when treated with emodin at $40 \mu \mathrm{M}$ for 24 or $48 \mathrm{~h}$ $(\mathrm{P}<0.01)$ (data not shown). Therefore, in this study, the cells were treated with emodin at 10 and $20 \mu \mathrm{M}$.

AR42J cells were divided into control, model (treated with cerulein plus LPS) and emodin treatment (treated with cerulein, LPS and emodin at 10 or $20 \mu \mathrm{M}$ ) groups. For the treatment groups, cells were pre-treated with emodin at various concentrations for $10 \mathrm{~min}$ prior to the addition of cerulein and LPS. After $3 \mathrm{~h}$ the culture media and cells were harvested for further analysis.

Measurement of amylase activity. Culture media was collected and the cells were washed twice with Krebs-HEPES buffer [140 mM NaCl, $5.9 \mathrm{mM} \mathrm{KCl}, 1.2 \mathrm{mM} \mathrm{MgCl}_{2}, 15 \mathrm{mM}$ HEPES, $10 \mathrm{mM}$ glucose, $2.5 \mathrm{mM} \mathrm{CaCl}_{2}, 0.1 \mathrm{mM}$ PMSF and $2.5 \mathrm{mM}$ aprotinin ( $\mathrm{pH}$ 7.4)]. Cells were then lysed by being forced to pass through a $26 \mathrm{G}$ syringe followed by brief sonication. The amylase activity in the culture media and cellular lysates was measured using an enzyme assay kit (Nanjing Jiancheng Bioengineering Institute, Nanjing, China). Amylase release was determined as the percentage of total amylase secreted into the culture media.

Measurement of cytosolic calcium. Fluorescence imaging was performed to evaluate the cytosolic calcium concentration. AR42J cells were plated at a density of $2 \times 10^{4}$ cells $/ \mathrm{cm}^{2}$ in 35-mm glass bottom dishes. Cells were loaded with $5 \mu \mathrm{M}$ membrane-permeable calcium indicator, Fluo-3AM ester, for $30 \mathrm{~min}$ at $37^{\circ} \mathrm{C}$ in Hank's buffer. Following loading with the fluo-3 dye, cells were washed with D-Hank's solution that lacked calcium and contained $5 \mathrm{mM}$ EGTA. Fluorescence measurements were performed using an Olympus Fluoview-500 confocal system equipped with a 40x objective (Olympus Corporations, Tokyo, Japan). Fluo-3 was excited by argon laser light at $488 \mathrm{~nm}$ wavelength and fluorescence was observed at $515 \mathrm{~nm}$ wavelength. Image processing and analysis were performed using Olympus Fluoview software. Intracellular calcium were expressed as average fluorescence intensity (FI).

Reverse transcription-polymerase chain reaction (RT-PCR). Gene expression of Bip, PERK, ATF6 and IRE1 $\alpha$ in AR42J cells was assessed using RT-PCR standardized by co-amplifying with GAPDH, which served as an internal control. Total RNAs isolated from the cells were reversely transcribed into cDNAs and used for PCR with rat specific primers for Bip, PERK, ATF6, IRE1 $\alpha$ and GAPDH. The sequences of primers used in the PCR were as follows: Forward: ATTCCTGCGTC GGTGTATT and reverse: TCGGCAGTTTCCTTCATTT for Bip; forward: TACAGTGGACGGCGATGATGAG and reverse: CTTAGGGTGGTTGGCCTGGTAG for PERK; forward: TCCCTCCACCTCCATGTCA and reverse: CTTCCAGGCGAAGCGTAAT for ATF6; forward: GCTGTGGAGACCCTACGCTAT and reverse: TCGATGTTTGGGAAGATTGTTAG for IRE1 $\alpha$; and forward, AAGGTCGGTGTGAACGGATTT and reverse, AGATGATGACCCTTTTGGCCC for GAPDH. The predicted sizes of the PCR products were: Bip, 358 bp; PERK, 270 bp; ATF6, 265 bp; IRE1 $\alpha, 426$ bp; and GAPDH, 352 bp. Following co-amplification with 32-35 cycles, the PCR products were separated on $1.5 \%$ agarose gels and visualized by UV transillumination.

Flow cytometry. AR42J cells were collected to detect apoptosis and necrosis using Annexin V-fluorescein isothiocyanate (FITC)/propidium iodide (PI) staining according to the manufacturer's instructions. Briefly, $1 \times 10^{5}$ cells were digested, 


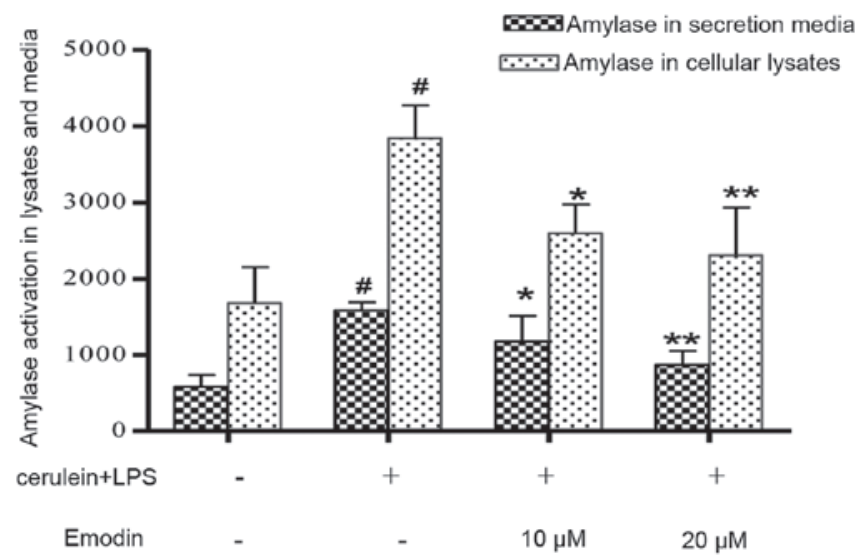

Figure 1. Effects of emodin on the expression and release of amylase in AR42J cells treated with cerulein plus LPS. Data are from three independent experiments and expressed as the mean $\pm \mathrm{SD} .{ }^{*} \mathrm{P}<0.01$, vs. control group; ${ }^{*} \mathrm{P}<0.05$ and ${ }^{* *} \mathrm{P}<0.01$, vs. model group. LPS, lipoplysaccharide.
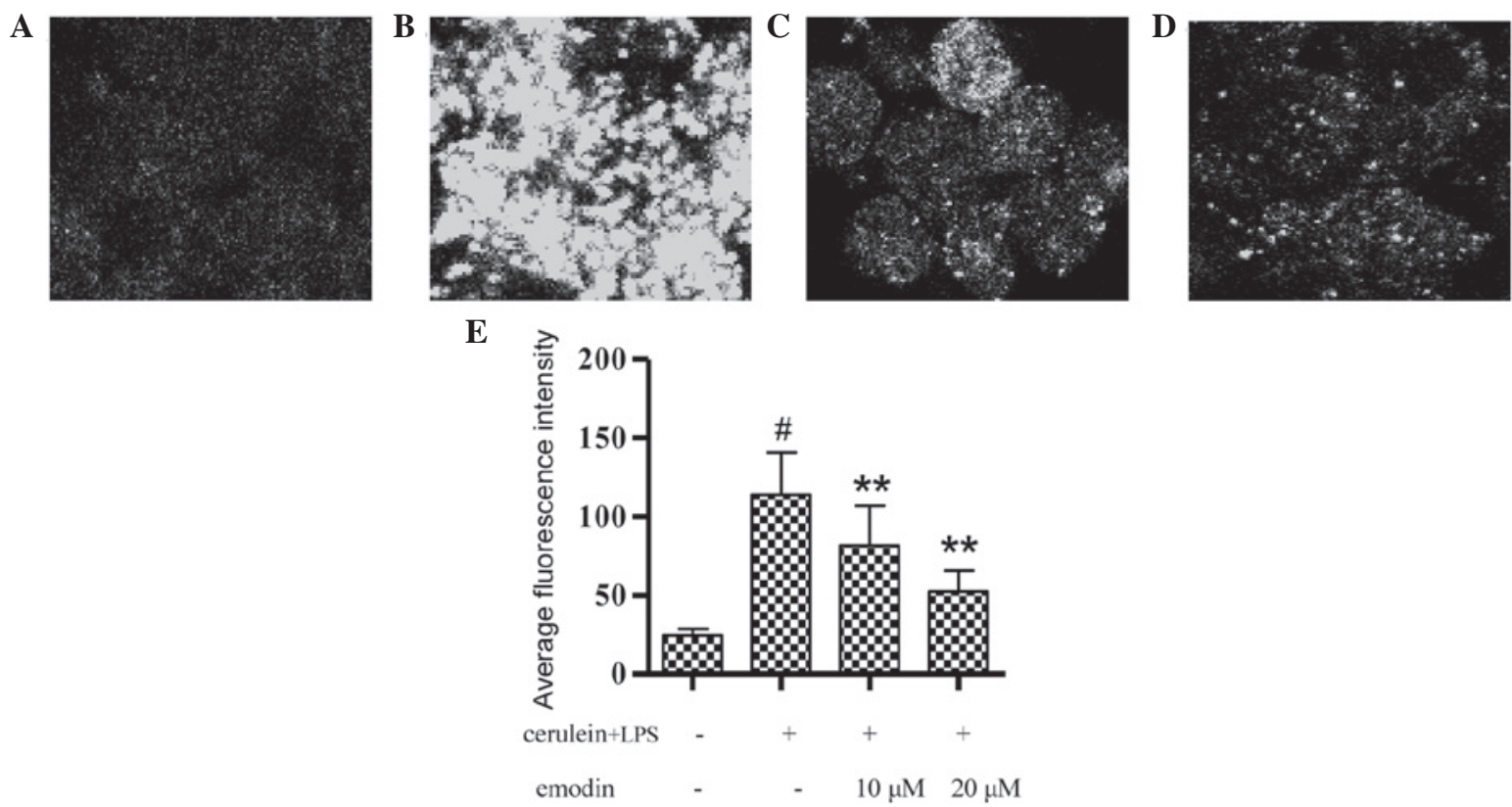

Figure 2. Effects of emodin on cytosolic calcium concentration in AR42J cells treated with cerulein. Data are from three independent experiments and expressed as the mean \pm standard deviation. ${ }^{*} \mathrm{P}<0.01$, vs. control group; ${ }^{*} \mathrm{P}<0.05$ and ${ }^{* * *} \mathrm{P}<0.01$, vs. the model group.

washed twice with cold PBS and re-suspended in $500 \mu 11 \mathrm{X}$ binding buffer. Next, $5 \mu 1$ annexin $\mathrm{V}$ and $10 \mu \mathrm{l}$ PI were added. Cells were gently vortexed and incubated for $15 \mathrm{~min}$ at $25^{\circ} \mathrm{C}$ in the dark followed by analysis by flow cytometry within $1 \mathrm{~h}$.

Statistical analysis. Data are presented as the mean \pm SD. The comparisons between two groups were performed by a one-way analysis of variance test. All statistical analyses were performed using SPSS 15.0 (SPSS, Inc., Chicago, IL, USA). $\mathrm{P}<0.05$ was considered to indicate a statistically significant difference.

\section{Results}

Effects of emodin on the expression and release of amylase in AR42J cells treated with cerulein and LPS. Effects of emodin on the expression and release of amylase are shown in Fig. 1. Compared with the control cells, the levels of amylase in cellular lysates and culture media were significantly elevated in the model group $(\mathrm{P}<0.01)$. Pretreatment with emodin markedly decreased the amylase activity in AR42J cell lysates and culture media.

Effects of emodin on cytosolic calcium concentration in AR42J cells treated with cerulein and LPS. The cytosolic calcium concentrations of AR42J cells are shown in Fig. 2. Stimulation of AR42J cells with cerulein plus LPS for $3 \mathrm{~h}$ led to calcium overload in the cytoplasm (Fig. 2B) and the average FI was higher than that of the control group (Fig. 2E; $\mathrm{P}<0.01$ ). Pretreatment with emodin at 10 or $20 \mu \mathrm{M}$ markedly alleviated calcium overload in AR42J cells (Fig. 2C and D) and also decreased the average FI value (Fig. 2F; P $<0.01$ ).

Effects of emodin on the mRNA expression of Bip, PERK, ATF6 and IRE1 in AR42J cells treated with cerulein plus $L P S$. UPR is a cellular response associated with ER stress. The 

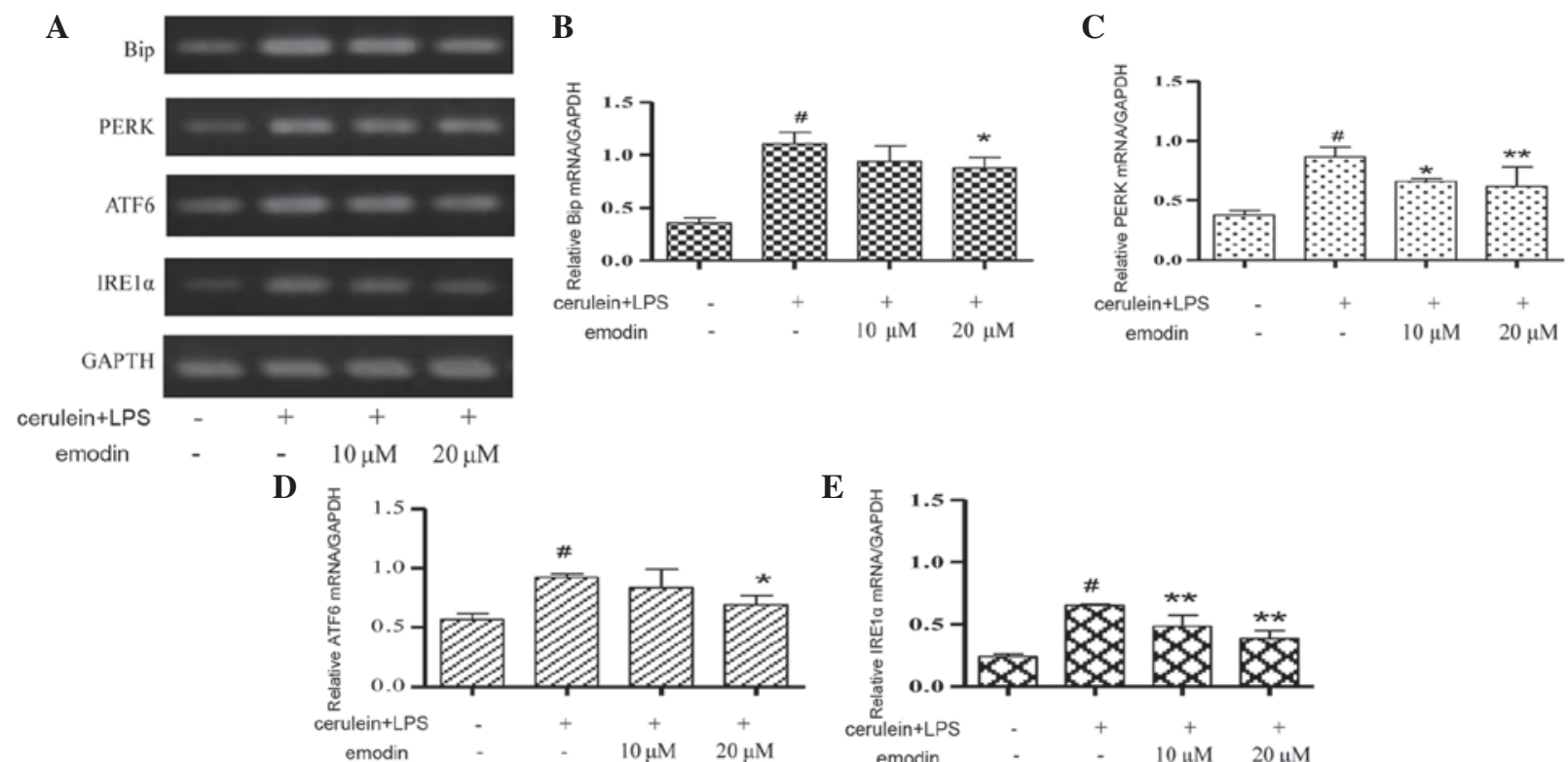

Figure 3. Effects of emodin on the mRNA expression of Bip, PERK, ATF6, and IRE1 in AR42J cells treated with cerulein plus LPS. Equal quantities of cell lysates were subjected to RT-PCR assay. GAPDH was used as an internal control. Fold changes in the mRNA levels of target genes associated with the invariant control GAPDH were calculated. Data are from three independent experiments and expressed as mean \pm standard deviation. ${ }^{*} \mathrm{P}<0.01$, vs. the control group; ${ }^{*} \mathrm{P}<0.05$ and $^{* *} \mathrm{P}<0.01$, vs. the model group. Bip, ER chaperone immunoglobulin-binding protein; PERK, protein kinase-like ER kinase; ATF6, activation transcription factor 6; IRE1, inositol-requiring protein 1.
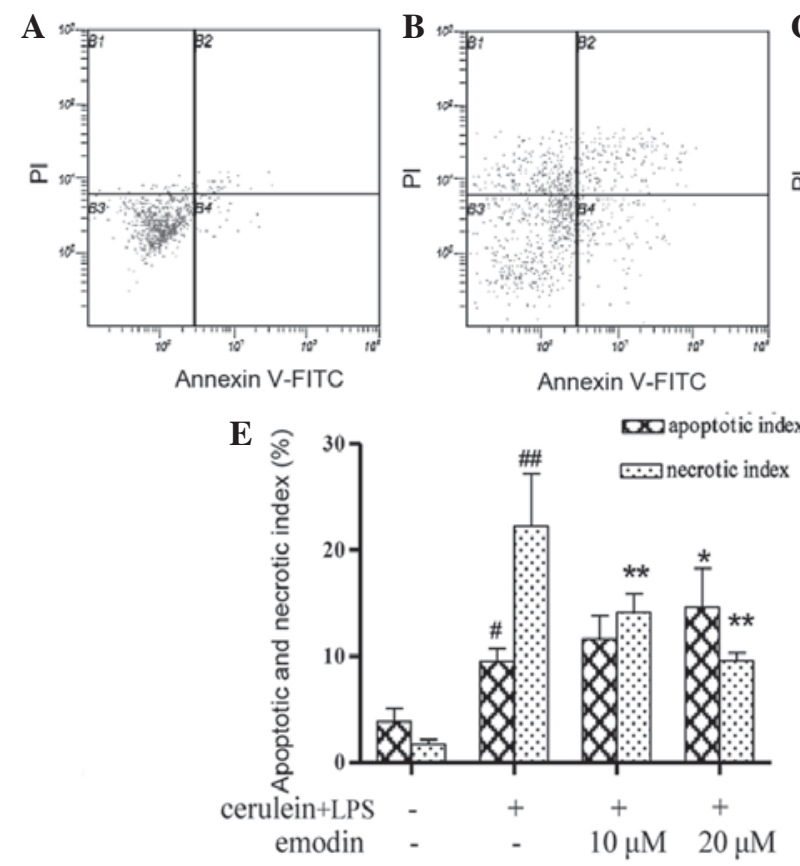

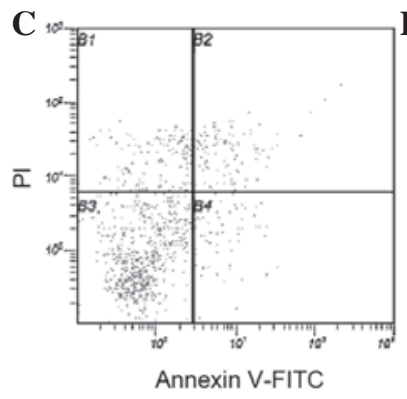

F

(1)
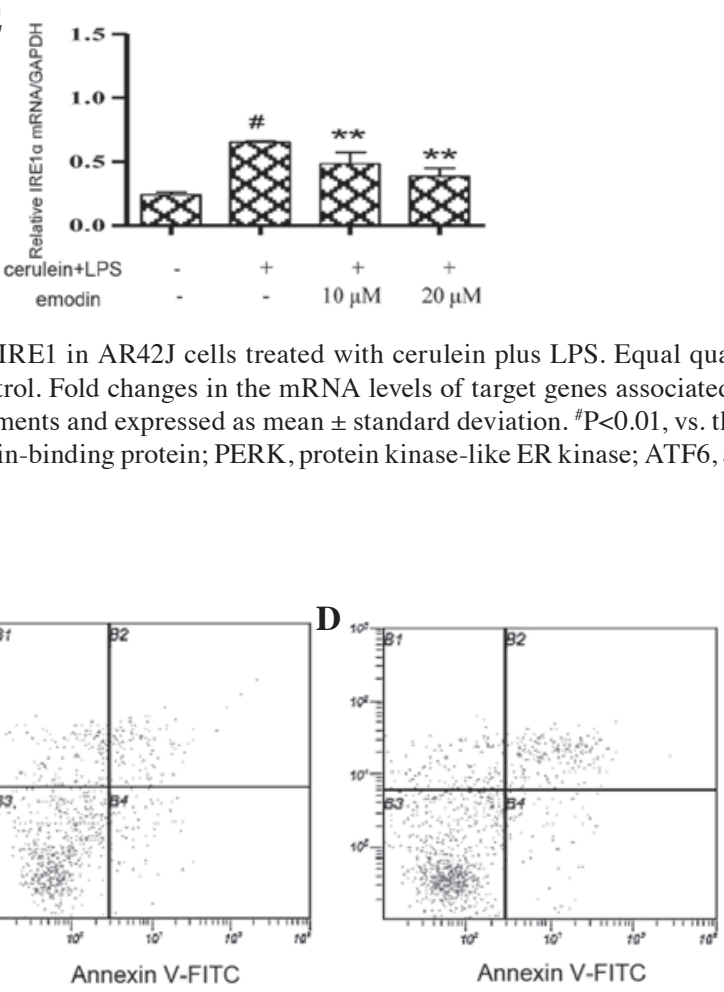

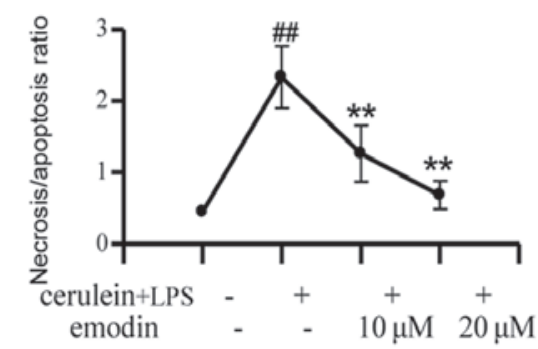

Figure 4. Effects of emodin on necrosis and apoptosis of AR42J cells treated with cerulein plus LPS. Flow cytometric analysis of AR42J cells stained with Annexin V-FITC and PI. Apoptotic cells are presented in the right-lower (B4) and right-upper (B2) quadrant of the plots; necrotic cells in the right-upper quadrant (B1); living cells in the left-lower quadrant (B3). Data are from 3 independent experiments and expressed as the mean \pm standard deviation. ${ }^{\#} \mathrm{P}<0.05$ and ${ }^{\# \#} \mathrm{P}<0.01$, vs. the control group; ${ }^{*} \mathrm{P}<0.05$ and ${ }^{* *} \mathrm{P}<0.01$, vs. the model group. LPS, lipoplysaccharide; FITC, fluorescein isothiocyanate; PI, propidium iodide.

mRNA expression of UPR transducers, including Bip, PERK, ATF6 and IRE1 in AR42J cells is shown in Fig. 3. In the model group, several UPR transducers were activated, as shown by the significantly increased mRNA expression of Bip, PERK, IRE1 and ATF6 compared with the control cells $(\mathrm{P}<0.01)$. Pretreatment with emodin at $10 \mu \mathrm{M}$ significantly reduced the mRNA expression of PERK $(\mathrm{P}<0.05)$ and IRE1 $(\mathrm{P}<0.01)$ and in the $20 \mu \mathrm{M}$ group, emodin significantly attenuated the upregulated mRNA levels of ER stress transducers, Bip $(\mathrm{P}<0.05)$, PERK $(\mathrm{P}<0.01)$, IRE1 $(\mathrm{P}<0.01)$ and ATF6 $(\mathrm{P}<0.05)$.

Effects of emodin on apoptosis and necrosis of AR42J cells treated with cerulein plus LPS. The occurrence of apoptosis and necrosis of AR42J cells is shown in Fig. 4. Compared 
with the control group, cerulein plus LPS caused a significant increase in apoptosis and necrosis of AR42J cells (Fig. 4A and $\mathrm{B})$. The apoptotic $(\mathrm{P}<0.05)$ and necrotic indices $(\mathrm{P}<0.01)$ were markedly increased (Fig. 4E). In addition, the necrosis/apoptosis ratio in the cerulein plus LPS group was 5 -fold higher than that in the control group (Fig. 4F). However, pretreatment with emodin at 10 or $20 \mu \mathrm{M}$ significantly attenuated the necrotic indices (Fig. 4C-E; P<0.01) and in the $20 \mu \mathrm{M}$ dose group, the apoptotic indices were increased (Fig. 4D and $\mathrm{E} ; \mathrm{P}<0.05)$. Accordingly, the necrosis/apoptosis ratio in AR42J cells treated with emodin was significantly lower than that in the model group (Fig. 4F; $\mathrm{P}<0.01$ ).

\section{Discussion}

The pancreatic acinar cell is the functional unit of the exocrine pancreas and synthesizes, stores and secretes digestive enzymes. Under physiological conditions, secretagogue substances, including cholecystokinin and acetylcholine, induce instantaneous increases in the concentration of cytosolic calcium, which passes through the plasma membrane and is released from the ER, in acinar cells. These processes are tightly controlled in a spatiotemporal manner and coupled to the physiological dose of zymogen expression (20). However, under pathological conditions, cytosolic calcium homeostasis is disrupted, as calcium stores (ER and apical stores) become depleted and the level of calcium in the cytoplasm remains elevated. To date, cytosolic calcium overload has been hypothesized to be pivotal in inducing pancreatic acinar cell injury (21), which was found to impair cellular defense mechanisms and initiate the activation of premature digestive enzymes within the cell. These processes led to the death of acinar cells $(22,23)$ with spillage of activated enzymes into the interstitial space, affecting the surrounding acinar cells and initiating a vicious circle that ends in AP and systemic inflammatory response syndrome. Thus, inhibition of calcium overload in the early stages of the disease is a valid avenue of therapeutic intervention for acinar cell injury and pancreatitis.

As an effective laxative, antiphlogistic and homeostatic traditional Chinese medicine, rhubarb is widely used in the treatment of digestive system diseases, including constipation, diarrhea, jaundice, pancreatitis and colitis (24). Emodin, a major ingredient isolated from rhubarb, has shown therapeutic promise for pancreatitis in vivo and in vitro, as it was found to suppress inflammation, promote apoptosis, inhibit pancreatin activity and scavenge oxygen free radicals. However, to date, few studies have determined whether emodin affects intracellular calcium homeostasis in pancreatic acinar cells during the development of pancreatitis, although specific studies have demonstrated that the anti-inflammatory and analgesic activities of emodin are associated with the regulation of intracellular calcium homeostasis.

Kuo et al (25) showed that emodin reduces the local inflammatory response by blocking calcium mobilization and diminishing inflammatory $\mathrm{T}$ cell activation and inflammatory cytokine production in rats with arthritis. Sui et al (26) reported that emodin inhibits inflammatory hyperalgesia by significantly downregulating the intracellular calcium fluorescence intensity in the dorsal root ganglion neurons and TRPV1
mRNA expression. In the present study, the effects of emodin on calcium overload were evaluated in AR42J cells treated with cerulein plus LPS, an in vitro model mimicking severe AP (27). The results showed that the levels of calcium in the cytoplasm remained elevated, accompanied by marked apoptosis and necrosis. However, pretreatment with emodin was found to markedly alleviate calcium overload in AR42J cells. Furthermore, emodin significantly ameliorated pancreatic cell injury by inducing apoptosis and decreasing necrosis.

The association between calcium overload and organelle dysfunction is being increasingly studied. As the most important reservoir of calcium, the ER participates in calcium overload during cell injury. Pathological stimuli, including fatty acid ethyl esters, cholecystokinin and cerulein, have been shown to induce massive calcium release via inositol triphosphate receptors from ER calcium stores, which is sustained by calcium entry resulting in mitochondrial depolarization, failure of ATP production and cellular necrosis (28). By contrast, calcium depletion of the ER may induce the perturbation of ER homeostasis leading to the activation of UPR that is associated with acinar cell injury, as well as death. As part of the ER stress responses, unfolded protein response contains at least three distinct signaling components that are activated, PERK, ATF6 and IRE1. These stress sensors and their downstream signaling partners participate in apoptosis and the inflammatory reaction when severe ER stress occurs (29). Severe stress also induces the release of ER calcium, which may stimulate mitochondrial generation of reactive oxygen species and lead to the activation of nuclear factor- $\kappa \mathrm{B}$, subsequently inducing inflammatory responses. Thus, sustained calcium overload and severe ER stress responses lead to a vicious cycle of cell injury and death.

In the present study, the effects of emodin on mRNA expression of the key stress sensors were investigated in AR42J cells using RT-PCR. Cerulein plus LPS-induced AR42J cell injury was accompanied by a significant increase in the mRNA expression of Bip, PERK, ATF6 and IRE1. Pretreatment with emodin reduced the mRNA expression of these molecules, particularly IRE1, whose mRNA level was significantly lowered by emodin at 10 and $20 \mu \mathrm{M}$ compared with the model cells, indicating that emodin attenuates ER stress responses in AR42J cells. IRE1 has been hypothesized to be an ER stress sensor protein and is important for the transduction of stress signals from the ER to the cytoplasm and nucleus (30). It has been shown that activated IRE1 $\alpha$ on the ER membrane recruits tumor necrosis factor receptor-associated factor 2 (TRAF2) and apoptosis signal-regulating kinase 1 (31). In addition, the activation of the IRE1 $\alpha /$ TRAF2 complex may result in the phosphorylation of c-Jun N-terminal kinase and p38 mitogen-activated protein kinases and promote pro-inflammatory cytokine activation, subsequently inducing inflammatory responses, cell injury and death $(32,33)$.

In conclusion, the results of the present study showed that calcium overload and ER stress are early molecular events of specific forms of cell injury, apoptosis and necrosis in AR42J cells. Emodin may alleviate cell injury and decrease the number of apoptotic and necrosis cells accompanied by increasing apoptosis and decreasing necrosis. These effects are likely to be associated with reduced calcium overload and suppressed ER stress responses. 


\section{Acknowledgements}

This work was supported by Priority Academic Program Development of Jiangsu Higher Education Institutions (ysxk-2010), Open Project Program of National First-Class Key Discipline for Traditional Chinese Medicine of Nanjing University of Chinese Medicine (2011ZYX4-006), National Natural Science Foundation of China (81073022), Science and Technology Foundation of Jiangsu Traditional Chinese Medicine Administration (LZ11190).

\section{References}

1. Talukdar R and Swaroop Vege S: Early management of severe acute pancreatitis. Curr Gastroenterol Rep 13: 123-130, 2011.

2. Kylänpää ML, Repo H and Puolakkainen PA: Inflammation and immunosuppression in severe acute pancreatitis. World J Gastroenterol 16: 2867-2872, 2010.

3. Gorelick FS and Thrower E: The acinar cell and early pancreatitis responses. Clin Gastroenterol Hepatol 7 (11 Suppl): S10-S14, 2009.

4. Kim JY, Kim KH, Lee JA, Namkung W, Sun AQ, Ananthanarayanan M, Suchy FJ, Shin DM, Muallem S and Lee MG: Transporter-mediated bile acid uptake causes $\mathrm{Ca}^{2+}$-dependent cell death in rat pancreatic acinar cells. Gastroenterology 122: 1941-1953, 2002.

5. Criddle DN, Raraty MG, Neoptolemos JP, Tepikin AV, Petersen $\mathrm{OH}$ and Sutton R: Ethanol toxicity in pancreatic acinar cells: mediation by nonoxidative fatty acid metabolites. Proc Natl Acad Sci USA 101: 10738-10743, 2004.

6. Criddle DN, Murphy J, Fistetto G, Barrow S, Tepikin AV, Neoptolemos JP, Sutton R and Petersen OH: Fatty acid ethyl esters cause pancreatic calcium toxicity via inositol trisphosphate receptors and loss of ATP synthesis. Gastroenterology 130 781-793, 2006.

7. Kubisch $\mathrm{CH}$ and Logsdon CD: Endoplasmic reticulum stress and the pancreatic acinar cell. Expert Rev Gastroenterol Hepatol 2: 249-260, 2008

8. Kubisch CH, Sans MD, Arumugam T, Ernst SA, Williams JA and Logsdon CD: Early activation of endoplasmic reticulum stress is associated with arginine-induced acute pancreatitis. Am J Physiol Gastrointest Liver Physiol 291: G238-G245, 2006.

9. Kubisch $\mathrm{CH}$ and Logsdon CD: Secretagogues differentially activate endoplasmic reticulum stress responses in pancreatic acinar cells. Am J Physiol Gastrointest Liver Physiol 292: G1804-G1812, 2007.

10. Seyhun E, Malo A, Schäfer C, Moskaluk CA, Hoffmann RT, Göke B and Kubisch CH: Tauroursodeoxycholic acid reduces endoplasmic reticulum stress, acinar cell damage, and systemic inflammation in acute pancreatitis. Am J Physiol Gastrointest Liver Physiol 301: G773-G782, 2011.

11. Szmola R and Sahin-Tóth M: Pancreatitis-associated chymotrypsinogen C (CTRC) mutant elicits endoplasmic reticulum stress in pancreatic acinar cells. Gut 59: 365-372, 2010.

12. Malo A, Krüger B, Seyhun E, Schäfer C, Hoffmann RT, Göke B and Kubisch CH: Tauroursodeoxycholic acid reduces endoplasmic reticulum stress, trypsin activation, and acinar cell apoptosis while increasing secretion in rat pancreatic acini. Am J Physiol Gastrointest Liver Physiol 299: G877-G886, 2010.

13. Ye R, Mareninova OA, Barron E, Wang M, Hinton DR, Pandol SJ and Lee AS: Grp78 heterozygosity regulates chaperone balance in exocrine pancreas with differential response to cerulein-induced acute pancreatitis. Am J Pathol 177: 2827-2836, 2010.

14. Wang G, Sun B, Gao Y, Meng QH and Jiang HC: The effect of emodin-assisted early enteral nutrition on severe acute pancreatitis and secondary hepatic injury. Mediators Inflamm 2007: 29638, 2007.
15. Li Z, Xia X, Zhang S, Zhang A, Bo W and Zhou R: Up-regulation of Toll-like receptor 4 was suppressed by emodin and baicalin in the setting of acute pancreatitis. Biomed Pharmacother 63: $120-128,2009$.

16. Xia XM, Li BK, Xing SM and Ruan HL: Emodin promoted pancreatic claudin-5 and occluding expression in experimental acute pancreatitis rats. World J Gastroenterol 18: 2132-2139, 2012.

17. Yu JH, Lim JW, Kim KH, Morio T and Kim H: NADPH oxidase and apoptosis in cerulein-stimulated pancreatic acinar AR42J cells. Free Radic Biol Med 39: 590-602, 2005.

18. Wu L, Cai B, Zheng S, Liu X, Cai H and Li H. Effect of emodin on endoplasmic reticulum stress in rats with severe acute pancreatitis. Inflammation 36: 1020-1029, 2013.

19. Eum WS, Li MZ, Sin GS, Choi SY, Park JB, Lee JY and Kwon HY: Dexamethasone-induced differentiation of pancreatic AR42J cell involves $\mathrm{p} 21$ (waf1/cip1) and MAP kinase pathway Exp Mol Med 35: 379-384, 2003

20. Low JT, Shukla A and Thorn P: Pancreatic acinar cell: new insights into the control of secretion. Int J Biochem Cell Biol 42: 1586-1589, 2010.

21. Frick TW: The role of calcium in acute pancreatitis. Surgery 152 (Suppl 1): S157-S163, 2012.

22. Criddle DN, Gerasimenko JV, Baumgartner HK, Jaffar M, Voronina S, Sutton R, Petersen $\mathrm{OH}$ and Gerasimenko OV: Calcium signalling and pancreatic cell death: apoptosis or necrosis? Cell Death Differ 14: 1285-1294, 2007.

23. Criddle DN, Sutton R and Petersen $\mathrm{OH}$ : Role of $\mathrm{Ca}^{2+}$ in pancreatic cell death induced by alcohol metabolites. J Gastroenterol Hepatol 21 (Suppl 3): S14-S17, 2006.

24. Chen DC and Wang L: Mechanisms of therapeutic effects of rhubarb on gut origin sepsis. Chin J Traumatol 12: 365-369, 2009.

25. Kuo YC, Meng HC and Tsai WJ: Regulation of cell proliferation, inflammatory cytokine production and calcium mobilization in primary human T lymphocytes by emodin from Polygonum hypoleucum Ohwi. Inflamm Res 50: 73-82, 2001.

26. Sui F, Huo HR, Zhang CB, Yang N, Guo JY, Du XL, Zhao BS, Liu HB, Li LF, Guo SY and Jiang TL: Emodin down-regulates expression of TRPV1 mRNA and its function in DRG neurons in vitro. Am J Chin Med 38: 789-800, 2010.

27. Liu Y, Zhou ZG, Chen KL, Zhou B, Yang L, Yan H and Li Y: The ER chaperone GRP78 is associated with the severity of cerulein-induced pancreatic inflammation via regulating apoptosis of pancreatic acinar cells. Hepatogastroenterology 59: 1670-1676, 2012.

28. Petersen $\mathrm{OH}$ and Sutton $\mathrm{R}: \mathrm{Ca}^{2+}$ signalling and pancreatitis: effects of alcohol, bile and coffee. Trends Pharmacol Sci 27: 113-120, 2006.

29. Kubisch $\mathrm{CH}$ and Logsdon CD: Secretagogues differentially activate endoplasmic reticulum stress responses in pancreatic acinar cells. Am J Physiol Gastrointest Liver Physiol 292: G1804-G1812, 2007.

30. Pavitt GD and Ron D: New insights into translational regulation in the endoplasmic reticulum unfolded protein response. Cold Spring Harb Perspect Biol 4: a012278, 2012.

31. Sendler M, Dummer A, Weiss FU, Krüger B, Wartmann T, Scharffetter-Kochanek K, van Rooijen N, Malla SR, Aghdassi A, Halangk W, Lerch MM and Mayerle J: Tumour necrosis factor $\alpha$ secretion induces protease activation and acinar cell necrosis in acute experimental pancreatitis in mice. Gut 62: 430-439, 2013.

32. Maguire JA, Mulugeta S and Beers MF: Endoplasmic reticulum stress induced by surfactant protein $\mathrm{C}$ Brichos mutants promotes proinflammatory signaling by epithelial cells. Am J Respir Cell Mol Biol 44: 404-414, 2011.

33. Kim SM, Chung MJ, Ha TJ, Choi HN, Jang SJ, Kim SO, Chun MH, Do SI, Choo YK and Park YI: Neuroprotective effects of black soybean anthocyanins via inactivation of ASK1-JNK/p38 pathways and mobilization of cellular sialic acids. Life Sciences 90: 874-882, 2012. 